\title{
Decomposition of Triply Rooted Trees
}

\author{
William Y. C. Chen ${ }^{1}$, Janet F. F. Peng ${ }^{2}$ and Harold R. L. Yang ${ }^{3}$ \\ ${ }^{1,2,3}$ Center for Combinatorics, LPMC-TJKLC \\ Nankai University, Tianjin 300071, P.R. China \\ ${ }^{1}$ Center for Applied Mathematics \\ Tianjin University, Tianjin 300072, P.R. China \\ ${ }^{1}$ chen@nankai.edu.cn, ${ }^{2}$ janet@mail.nankai.edu.cn \\ 3 yangruilong@mail.nankai.edu.cn
}

Submitted: Dec 28, 2012; Accepted: Apr 9, 2013; Published: Apr 17, 2013

Mathematics Subject Classifications: 05A15, 05A19

\begin{abstract}
We give a decomposition of triply rooted trees into three doubly rooted trees. This leads to a combinatorial interpretation of an identity conjectured by Lacasse in the study of the PAC-Bayesian machine learning theory, and proved by Younsi by using the Hurwitz identity on multivariate Abel polynomials. Let $[n]=\{1,2, \ldots, n\}$. We also give a bijection between the set of functions from $[n+1]$ to $[n]$ and the set of triply rooted trees on $[n]$, which leads to a symmetry property and a refined enumeration of functions from $[n+1]$ to $[n]$ with respect to the number of elements in the orbit of $n+1$ and the number of periodic points.
\end{abstract}

Keywords: doubly rooted tree, triply rooted tree, bijection

\section{Introduction}

Lacasse [5] introduced the functions $\xi(n)$ and $\xi_{2}(n)$ in his study of the classical PAC-Bayes theorem in the theory of machine learning, where

$$
\xi(n)=\sum_{k=0}^{n}\left(\begin{array}{l}
n \\
k
\end{array}\right)\left(\frac{k}{n}\right)^{k}\left(1-\frac{k}{n}\right)^{n-k}
$$


and

$$
\xi_{2}(n)=\sum_{j=0}^{n} \sum_{k=0}^{n-j}\left(\begin{array}{c}
n \\
j
\end{array}\right)\left(\begin{array}{c}
n-j \\
k
\end{array}\right)\left(\frac{j}{n}\right)^{j}\left(\frac{k}{n}\right)^{k}\left(1-\frac{j}{n}-\frac{k}{n}\right)^{n-j-k} .
$$

He showed that $\xi(n)$ can be used to give a tighter bound of the Kullback-Leibler divergence between the risk and the empirical risk on a sample space $S$ of a hypothesis function in a hypothesis space, whereas $\xi_{2}(n)$ can be used to bound the Kullback-Leibler divergence between the risk and the empirical risk on $S$ of the joint distribution of two hypothesis functions in a hypothesis space.

While $\xi_{2}(n)$ is a double sum, based on numerical evidence Lacasse [5] posed the following conjecture stating that $\xi_{2}(n)$ can be reduced to the single sum $\xi(n)$.

Conjecture 1.1 For $n \in \mathbb{N}$, we have

$$
\xi_{2}(n)=\xi(n)+n
$$

By applying a multivariate Abel identity due to Hurwitz, Younsi [9] gave an algebraic proof of this conjecture. Let

$$
A_{n}\left(x_{1}, x_{2}, \ldots, x_{m} ; p_{1}, p_{2}, \ldots, p_{m}\right)=\sum_{k_{1}+k_{2}+\cdots+k_{m}=n}\left(\begin{array}{c}
n \\
k_{1}, k_{2}, \ldots, k_{m}
\end{array}\right) \prod_{j=1}^{m}\left(x_{j}+k_{j}\right)^{k_{j}+p_{j}}
$$

where $p_{1}, p_{2}, \ldots, p_{m}$ are integers. When $p_{1}=p_{2}=\cdots=p_{m}=0$, Hurwitz proved that

$$
A_{n}\left(x_{1}, \ldots, x_{m} ; 0, \ldots, 0\right)=\sum_{k=0}^{n}\left(\begin{array}{l}
n \\
k
\end{array}\right)\left(x_{1}+x_{2}+\cdots+x_{m}+n\right)^{n-k} \alpha_{k}(m-1),
$$

where $\alpha_{k}(r)=r(r+1) \cdots(r+k-1)$ is the rising factorial, see, for example, Riordan [7].

Younsi [9] observed that $\xi(n)=A_{n}(0,0 ; 0,0)$ and $\xi_{2}(n)=A_{n}(0,0,0 ; 0,0,0)$, and derived the following expressions for $\xi(n)$ and $\xi_{2}(n)$ by using $(1.2)$

$$
\begin{aligned}
\xi(n) & =\frac{1}{n^{n}} \sum_{j=0}^{n} n^{j} \frac{n !}{j !} \\
\xi_{2}(n) & =\frac{1}{n^{n}} \sum_{j=0}^{n} n^{n-j}\left(\begin{array}{l}
n \\
j
\end{array}\right)(j+1) !
\end{aligned}
$$

Conjecture 1.1 can be easily deduced from (1.3) and (1.4). Other proofs have been found by Prodinger [6] and Sun [8].

In this paper, we give a combinatorial explanation of relation (1.1). Rewriting (1.1) as

$$
\sum_{j=0}^{n} \sum_{k=0}^{n-j}\left(\begin{array}{c}
n \\
j
\end{array}\right)\left(\begin{array}{c}
n-j \\
k
\end{array}\right) j^{j} k^{k}(n-j-k)^{n-j-k}=\sum_{k=0}^{n}\left(\begin{array}{l}
n \\
k
\end{array}\right) k^{k}(n-k)^{n-k}+n^{n+1}
$$


we see that it is equivalent to the following form

$$
\sum_{j=1}^{n} \sum_{k=0}^{n-j}\left(\begin{array}{c}
n \\
j
\end{array}\right)\left(\begin{array}{c}
n-j \\
k
\end{array}\right) j^{j} k^{k}(n-j-k)^{n-j-k}=n^{n+1}
$$

The right hand side of (1.6) indicates that we need the notion of triply rooted trees, namely, labeled trees with an ordered list of three distinguished, but not necessarily distinct vertices. To be more specific, the three distinguished vertices of a triply rooted tree are called the first, the second and the third root, respectively. It can be easily seen that the summand on the left hand side of (1.6) can be interpreted as the number of triples of doubly rooted trees with a given number of vertices in each doubly rooted tree. Hence relation (1.6) can be deduced from a decomposition of a triply rooted tree into three doubly rooted trees.

The second result of this paper is a correspondence between the set of functions from $[n+1]$ to $[n]$ and the set of triply rooted trees on $[n]$, where $n$ is a positive integer and $[n]=\{1,2, \ldots, n\}$. Let $f$ be a function from $[n+1]$ to $[n]$ and let $T$ be the corresponding triply rooted tree. We find that the orbit of $n+1$ on $f$ is mapped to the set of ancestors of the second root in $T$, and the set of periodic points of $f$ is mapped to the set of ancestors of the third root in $T$. Based on this property of our bijection, we deduce a symmetry property of functions from $[n+1]$ to $[n]$ with respect to the number of periodic points and the size of the orbit of $n+1$. Moreover, we obtain a formula for the number of triply rooted trees for which the second root has $i$ ancestors and the third root has $j$ ancestors. This formula also gives the number of functions from $[n+1]$ to $[n]$ such that the size of the orbit of $n+1$ is $i+1$ and the number of periodic points is $j$.

\section{Decomposition of triply rooted tree}

In this section, we give a combinatorial interpretation of Lacasse's identity by providing a decomposition of a triply rooted tree into three doubly rooted trees.

Recall that a rooted tree is defined to be a labeled tree with a specific vertex, which is called the root. Let $R_{n}$ denote the set of rooted trees on $[n]$. It is well-known that the set $R_{n}$ is counted by $n^{n-1}$, as shown by Cayley [2]. A doubly rooted tree is defined as a labeled tree with an ordered list of two distinguished vertices $r_{1}$ and $r_{2}$, where we call $r_{1}$ the first root and $r_{2}$ the second root. Similarly, a triply rooted tree is a labeled tree with an ordered list of three distinguished vertices. Notice that the roots are not necessarily distinct. We denote by $D_{n}\left(T_{n}\right)$ the set of doubly (triply) rooted trees on $[n]$. One sees that $\left|D_{n}\right|=n^{n}$ and $\left|T_{n}\right|=n^{n+1}$. A bijection from doubly rooted trees to functions from $[n]$ to $[n]$ was independently obtained by Joyal [4] and Goulden and Jackson [3]. Hence the right hand side of (1.6) can be interpreted as the number of triply rooted trees on $[n]$. 
On the other hand, let $Q_{n}$ denote the set of triples of doubly rooted trees $\left(D, D^{\prime}, D^{\prime \prime}\right)$ such that the vertex sets of $D, D^{\prime}, D^{\prime \prime}$ form a weak partition of $[n]$ with $D$ being nonempty. To be more specific, a triple $(X, Y, Z)$ of subsets of a set $S$ is said to be a weak partition of $S$ if $X, Y$, and $Z$ are disjoint and their union equals $S$. It is obvious that $Q_{n}$ is counted by

$$
\sum_{j=1}^{n} \sum_{k=0}^{n-j}\left(\begin{array}{c}
n \\
j
\end{array}\right)\left(\begin{array}{c}
n-j \\
k
\end{array}\right) j^{j} k^{k}(n-j-k)^{n-j-k},
$$

which is the left hand side of (1.6). Hence identity (1.6) follows from the following bijection.

Theorem 2.1 For $n \geqslant 1$, there is a bijection between $Q_{n}$ and $T_{n}$.

To present the proof of the above theorem, we recall some terminology. Given two vertices $i$ and $j$ of a rooted tree $T$, we say that $j$ is a descendant of $i$, or $i$ is an ancestor of $j$, if $i$ lies on the unique path from the root to $j$. In particular, each vertex is a descendant as well as an ancestor of itself. A child of $i$ means a descendant $j$ of $i$ such that $(i, j)$ is an edge of $T$. The depth of $i$ is defined to be the number of edges of the unique path from the root to $i$. Given two vertices $v_{1}$ and $v_{2}$ of $T$, there is a unique vertex $v$ that is the common ancestor of $v_{1}$ and $v_{2}$ with the largest depth. This vertex is called the least common ancestor of $v_{1}$ and $v_{2}$, see Aho, Hopcroft and Ullman [1]. For example, for the tree in Figure 2.1, the least common ancestor of 1 and 3 is 5 , while the least common ancestor of 1 and 6 is the root 4 .

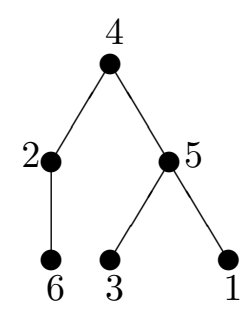

Figure 2.1: A rooted tree on [6].

Throughout this paper, we use $r_{1}(D)$ and $r_{2}(D)$ to denote the first root and the second root of a doubly rooted tree $D$, respectively, and we use $r_{1}(T), r_{2}(T)$ and $r_{3}(T)$ to denote the first root, the second root and the third root of a triply rooted tree $T$, respectively.

Proof of Theorem 2.1. We define a map $\varphi$ from $Q_{n}$ to $T_{n}$. Given a triple $\left(D, D^{\prime}, D^{\prime \prime}\right)$ of doubly rooted trees in $Q_{n}$, we first consider the case when neither $D^{\prime}$ nor $D^{\prime \prime}$ is empty.

We merge $D$ and $D^{\prime}$ by setting $r_{1}\left(D^{\prime}\right)$ to be a child of $r_{2}(D)$, and we merge $D$ and $D^{\prime \prime}$ by setting $r_{1}\left(D^{\prime \prime}\right)$ to be a child of $r_{2}(D)$. By setting $r_{2}\left(D^{\prime}\right)$ and $r_{2}\left(D^{\prime \prime}\right)$ to be the second root and the third root of the resulting tree, we obtain a triply rooted tree $T$. 
For example, Figure 2.2 gives an illustration of a triple of doubly rooted trees and the corresponding triply rooted tree, where the second root is represented by a solid square, and the third root is represented by a hollow square.
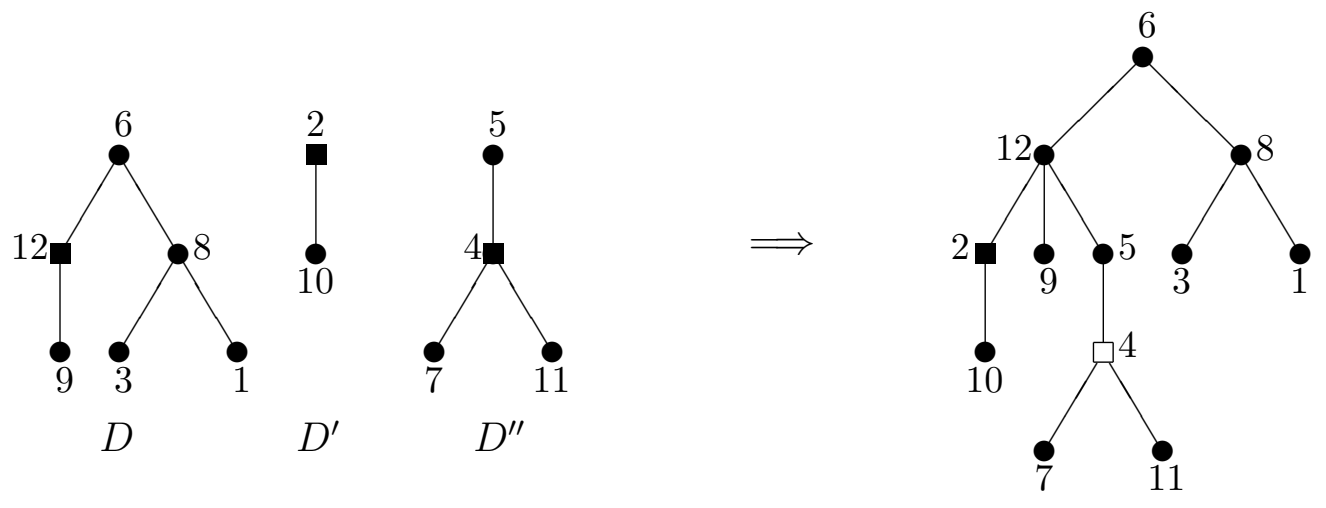

Figure 2.2: The merging process when $D^{\prime} \neq \emptyset$ and $D^{\prime \prime} \neq \emptyset$.

We now consider the case when either $D^{\prime}$ or $D^{\prime \prime}$ is empty. When $D^{\prime}=\emptyset$, there is no need to merge $D$ and $D^{\prime}$. Instead, we set $r_{2}(D)$ to be the second root of the resulting tree in the merging process. Similarly, when $D^{\prime \prime}=\emptyset$, we just set $r_{2}(D)$ to be the third root of the resulting tree in the merging process.

In summary, $\left(r_{1}(T), r_{2}(T), r_{3}(T)\right)$ is given as follows:

$$
\begin{cases}\left(r_{1}(D), r_{2}\left(D^{\prime}\right), r_{2}\left(D^{\prime \prime}\right)\right), & \text { if } D^{\prime} \neq \emptyset, \text { and } D^{\prime \prime} \neq \emptyset ; \\ \left(r_{1}(D), r_{2}(D), r_{2}\left(D^{\prime \prime}\right)\right), & \text { if } D^{\prime}=\emptyset, \text { and } D^{\prime \prime} \neq \emptyset ; \\ \left(r_{1}(D), r_{2}\left(D^{\prime}\right), r_{2}(D)\right), & \text { if } D^{\prime} \neq \emptyset, \text { and } D^{\prime \prime}=\emptyset \\ \left(r_{1}(D), r_{2}(D), r_{2}(D)\right), & \text { if } D^{\prime}=\emptyset, \text { and } D^{\prime \prime}=\emptyset\end{cases}
$$

Figure 2.3 gives an illustration of the merging process when $D^{\prime}=\emptyset$ and $D^{\prime \prime} \neq \emptyset$.

To show that the above process is invertible, we give a description of the inverse procedure. Given a triply rooted tree $T$ with three roots $r_{1}, r_{2}$ and $r_{3}$, assume that $w$ is the least common ancestor of $r_{2}$ and $r_{3}$.

We first consider the case when $w \neq r_{2}$ and $w \neq r_{3}$. Find the child $x$ of $w$ such that $r_{2}$ is a descendant of $x$, and the child $y$ of $w$ such that $r_{3}$ is a descendant of $y$. Removing the edges $(w, x)$ and $(w, y)$, we get three trees with three roots $r_{1}, x$ and $y$. Let $D$ be the doubly rooted tree with two roots $r_{1}$ and $w$, let $D^{\prime}$ be the doubly rooted tree with two roots $x$ and $r_{2}$, and let $D^{\prime \prime}$ be the doubly rooted tree with two roots $y$ and $r_{3}$.

When $w=r_{2}$, then in the previous process, $x$ does not exist, and instead we set $D^{\prime}=\emptyset$. Similarly, when $w=r_{3}$, we set $D^{\prime \prime}=\emptyset$. 

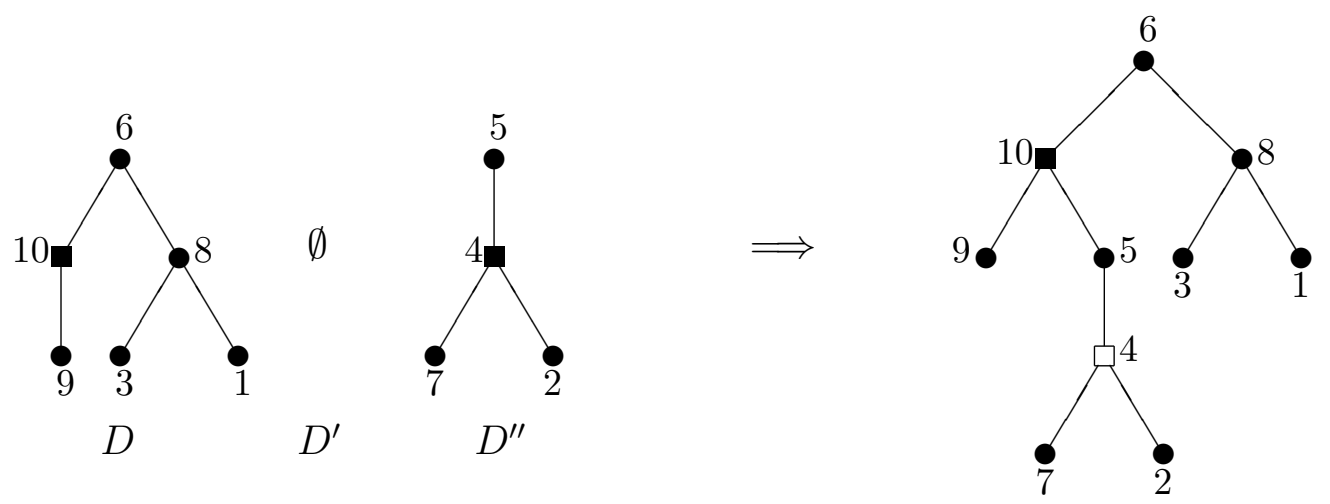

Figure 2.3: The merging process when $D^{\prime}=\emptyset$ and $D^{\prime \prime} \neq \emptyset$.

Now we see that in any case the three doubly rooted trees $D, D^{\prime}$ and $D^{\prime \prime}$ can be merged into the triply rooted tree $T$. That is, the above merging process is invertible. This completes the proof.

\section{Functions from $[n+1]$ to $[n]$}

In this section, we establish a correspondence between functions from $[n+1]$ to $[n]$ and triply rooted trees on $[n]$, which maps the orbit of $n+1$ to the set of ancestors of the second root, and maps the set of periodic points to the set of ancestors of the third root. By the symmetry between the second and third roots, we deduce a symmetry property of the number of functions from $[n+1]$ to $[n]$ with respect to the number of periodic points and the size of the orbit of $n+1$.

Given a function $f$ from $[n+1]$ to $[n]$, the orbit of $x$ on $f$ is defined to be the set $\left\{x, f(x), f^{2}(x), \ldots\right\}$. If there exists some $j \geqslant 1$, such that $f^{j}(x)=x$, then $x$ is called a periodic point of $f$. We have the following correspondence.

Theorem 3.1 There is a bijection $\phi$ between the set of functions $f$ from $[n+1]$ to $[n]$ and the set of triply rooted trees on $[n]$ such that the orbit of $n+1$ on $f$ excluding $n+1$ itself is mapped to the set of ancestors of the second root of $\phi(f)$ and the set of periodic points of $f$ is mapped to the set of ancestors of the third root of $\phi(f)$.

Proof. The map $\phi$ can be described as follows. Let $f$ be a function from $[n+1]$ to $[n]$. We proceed to construct a triply rooted tree $T$ on $[n]$ based on $f$. We begin with the functional digraph $G_{f}$ of $f$, that is, a digraph on $[n+1]$ with $\operatorname{arcs}(i, f(i))$ for $1 \leqslant i \leqslant n+1$. Let $C_{1}$ be the connected component of $G_{f}$ containing the vertex $n+1$. Consider the longest path $P$ starting from $n+1$, say,

$$
P: n+1=u_{0} \rightarrow u_{1} \rightarrow u_{2} \rightarrow \cdots \rightarrow u_{k} .
$$


In other words, $k$ is the smallest integer such that $f\left(u_{k}\right)$ equals $u_{j}$ for some $j \leqslant k$. Assume that $f\left(u_{k}\right)=u_{j}$. Removing the arc $\left(u_{k}, u_{j}\right)$ and the vertex $n+1$ from $C_{1}$, we get a tree $H$ rooted at $u_{k}$.

Let $C_{2}$ be the digraph $G_{f} \backslash C_{1}$. When $C_{2}=\emptyset$, we set $u_{k}, u_{1}$ and $u_{j}$ to be the three roots of $H$ to obtain a triply rooted tree $T$.

When $C_{2} \neq \emptyset$, we see that $C_{2}$ is a functional digraph. By applying the bijection between functions and doubly rooted trees, obtained by Joyal [4] and Goulden and Jackson [3], $C_{2}$ corresponds to a doubly rooted tree $D$. Let $w_{1}$ and $w_{2}$ be the two roots of $D$. We merge the rooted tree $H$ and the doubly rooted tree $D$ by setting the first root $w_{1}$ of $D$ as a child of the vertex $u_{j}$ of $H$. Setting $u_{k}, u_{1}$ and $w_{2}$ to be the first, the second and the third root, respectively, we get a triply rooted tree $T$, and we set $\phi(f)=T$.

For example, let $f$ be the following function from [13] to [12]

$$
f=\left(\begin{array}{ccccccccccccc}
1 & 2 & 3 & 4 & 5 & 6 & 7 & 8 & 9 & 10 & 11 & 12 & 13 \\
8 & 6 & 8 & 5 & 4 & 12 & 4 & 6 & 12 & 2 & 4 & 2 & 3
\end{array}\right) .
$$

The functional digraph of $f$ is given in Figure 3.4, where $C_{1}$ is the functional digraph on $\{1,2,3,6,8,9,10,12,13\}$ and $C_{2}$ is the functional digraph on $\{4,5,7,11\}$. The longest

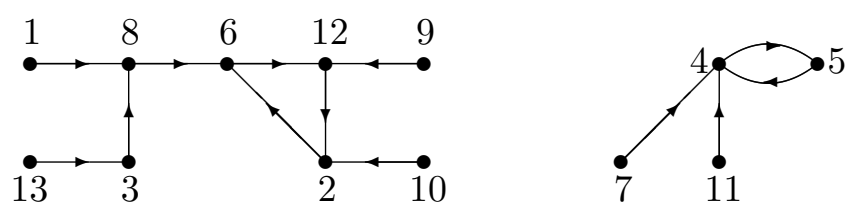

Figure 3.4: The functinal digraph $G_{f}$.

path starting from 13 is

$$
P: 13 \rightarrow 3 \rightarrow 8 \rightarrow 6 \rightarrow 12 \rightarrow 2,
$$

with $f(2)=6$, that is, $u_{1}=3, u_{k}=2$ and $u_{j}=6$ as in the proof. Deleting the arc $(2,6)$ and vertex 13, we get a rooted tree $H$ as illustrated in Figure 3.5. By applying the bijection between functional digraphs and doubly rooted trees, $C_{2}$ can be mapped to a doubly rooted tree $D$ with roots 5 and 4 as shown in Figure 3.5, where $w_{1}=5$ and $w_{2}=4$ as in the proof. Merging $H$ and $D$ by adding an edge $(6,5)$ and setting 2,3 and 4 to be the three roots, we get a triply rooted tree $T$ in Figure 3.5.

To prove that $\phi$ is a bijection, we give a description of the inverse map. For a triply rooted tree $T \in T_{n}$ with the three roots $r_{1}, r_{2}$ and $r_{3}$, we first find the least common ancestor of $r_{2}$ and $r_{3}$, and we denote it by $u_{0}$. Suppose that the unique path $P$ from $u_{0}$ to $r_{3}$ in $T$ is $u_{0} u_{1} \cdots u_{i}=r_{3}$. Removing the edge $\left(u_{0}, u_{1}\right)$ from $T$, we get two components $T_{1}$ and $T_{2}$, where $T_{1}$ is rooted at $r_{1}$ and $T_{2}$ is rooted at $u_{1}$. Adding $n+1$ to $T_{1}$ by setting it 


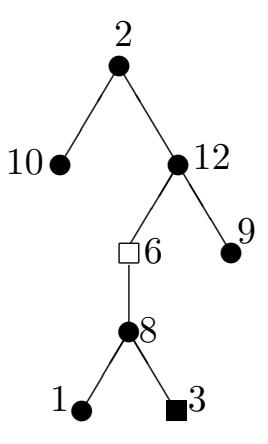

Tree $H$

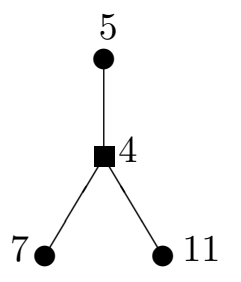

Tree $D$

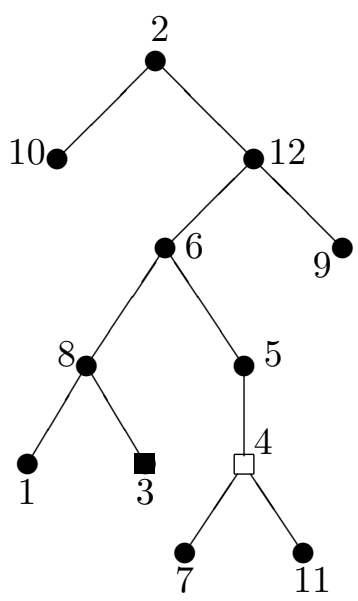

Tree $T$

Figure 3.5: An example of the bijection $\phi$.

as a child of $r_{2}$. Now, $T_{1}$ can be viewed as a directed graph by making each edge point to the father. Then we add the arc $\left(r_{1}, u_{0}\right)$ to $T_{1}$ to obtain a connected functional digraph $C_{1}$.

Next, we transform $T_{2}$ rooted at $u_{1}$ into a doubly rooted tree by setting $r_{3}$ to be the second root. Then we get a functional digraph $C_{2}$ by applying the inverse map of the bijection of Joyal [4] and Goulden and Jackson [3].

Finally, let $G=C_{1} \cup C_{2}$. Observe that $G$ is a directed graph on $[n+1]$ such that each vertex has outdegree one and the vertex $n+1$ has indegree zero. In other words, $G$ is the functional digraph of a function from $[n+1]$ to $[n]$. We see that the above procedure is the inverse of the map $\phi$.

It remains to prove the properties of $\phi$ as stated in the theorem. For a function $f$ from $[n+1]$ to $[n]$, an element $x$ is a periodic point in $f$ if and only if it is a vertex in a cycle in the functional digraph $G_{f}$. We see that $x$ is in a cycle if and only if it is an ancestor of the third root in the triply rooted tree $\phi(f)$. Moreover, we observe that each element $y$ in the orbit of $n+1$ on $f$ other than $n+1$ itself corresponds to an ancestor of the second root in the triply rooted tree $\phi(f)$. This completes the proof.

For example, for the function $f$ in Figure 3.4, there are five periodic points 2, 12, 6, 5, 4, which are the vertices in the path from the root 2 to the third root 4 in $\phi(f)$ as demonstrated in Figure 3.5. The orbit of 13 consists of $13,3,8,6,12,2$. The elements 3, 8, 6, 12, 2 correspond to the vertices in the path from the root 2 to the second root 3 in $\phi(f)$.

We notice that the bijection $\phi$ leads to a symmetry property of functions from $[n+1]$ to $[n]$. Let $W_{n, i, j}$ denote the set of triply rooted trees on $[n]$ such that the depth of the 
second root is $i$ and the depth of the third root is $j$. Let $F_{n, i, j}$ denote the set of functions from $[n+1]$ to $[n]$ such that the size of the orbit of $n+1$ is $i$ and the number of periodic points is $j$. By Theorem 3.1, we see that

$$
\left|F_{n, i+1, j}\right|=\left|W_{n, i-1, j-1}\right| \text {. }
$$

By the symmetry between the second roots and the third roots of triply rooted trees, that is,

$$
\left|W_{n, i, j}\right|=\left|W_{n, j, i}\right|,
$$

we arrive at the following symmetry property of functions from $[n+1]$ to $[n]$,

$$
\left|F_{n, i+1, j}\right|=\left|F_{n, j+1, i}\right| \text {. }
$$

The following theorem gives a formula for $W_{n, i, j}$.

Theorem 3.2 For $n \geqslant 1$ and $0 \leqslant i, j \leqslant n-1$, we have

$$
\left|W_{n, i, j}\right|=\sum_{d=0}^{\min \{i, j\}} \frac{(i+j-d+1) n !}{(n-i-j+d-1) !} n^{n-i-j+d-2},
$$

where $\min \{i, j\}$ denotes the smaller value between $i$ and $j$.

Proof. Let $W_{n, i, j}(d)$ denote the set of triply rooted trees $T$ in $W_{n, i, j}$ such that $d$ is the depth of the least common ancestor of the second root and the third root of $T$. We proceed to show that $W_{n, i, j}(d)$ is enumerated by the summand on the right hand side of $(3.2)$.

Let $T$ be a triply rooted tree in $W_{n, i, j}(d)$. We denote by $P_{1}$ the path from the first root to the second root and denote by $P_{2}$ the path from the first root to the third root. Observing that there are exactly $k=i+j-d+1$ vertices on $P_{1}$ and $P_{2}$, we see the number of ways to form $P_{1}$ and $P_{2}$ equals $\frac{n !}{(n-k) !}$. Moreover, as we know, there are $k n^{n-k-1}$ forests consisting of $k$ rooted trees on $[n]$ with $k$ given roots. It follows that $W_{n, i, j}(d)$ is enumerated by the summand on the right hand side of (3.3). This completes the proof.

Combining Theorem 3.1 and Theorem 3.2, we obtain the following formula for $\left|F_{n, i+1, j}\right|$.

Theorem 3.3 For $n \geqslant 1$ and $1 \leqslant i, j \leqslant n$, we have

$$
\left|F_{n, i+1, j}\right|=\sum_{s=0}^{\min \{i, j\}-1} \frac{(i+j-s-1) n !}{(n-i-j+s+1) !} n^{n-i-j+s} .
$$

Acknowledgments. We wish to thank Catherine H.F. Yan and the referee for valuable suggestions. This work was supported by the 973 Project, the PCSIRT Project of the Ministry of Education, and the National Science Foundation of China. 


\section{References}

[1] A.V. Aho, J.E. Hopcroft and J.D. Ullman, On finding lowest common ancestors in trees, SIAM J. Comput. 5 (1976), 115-132.

[2] A. Cayley, Note sur une formule pour la réversion des séries, J. reine angew. Math. 52 (1856), 276-284.

[3] I.P. Goulden and D.M. Jackson, Combinatorial Enumeration, John Wiley, New York, 1983.

[4] A. Joyal, Une théorie combinatoire des séries formelles, Adv. Math. 42 (1981), 1-82.

[5] A. Lacasse, Bornes PAC-Bayes et algorithmes d'apprentissage, Ph.D. Thesis, Universite Laval, Quebec, 2010.

[6] H. Prodinger, An identity conjectured by Lacasse via the tree function, arXiv:1301.3669.

[7] J. Riordan, Combinatorial Identities, Robert E. Krieger Publishing Co., New York, 1968.

[8] Y. Sun, A simple proof of an identity of Lacasse, Electron. J. Combin. 20(2) (2013), \#P11.

[9] M. Younsi, Proof of a combinatorial conjecture coming from the PAC-Bayesian machine learning theory, arXiv:1209.0824. 